\title{
TEOR DE CLOROFILA DE SEIS ESPÉCIES ARBÓREAS SOB INFLUÊNCIA DA POEIRA DE CIMENTO
}

\author{
Mario Takao Inoue ${ }^{1}$ \\ ${ }^{1}$ Eng. Florestal, Dr., Depto. de Engenharia Florestal, UNICENTRO, Irati, PR, Brasil - takao@irati.unicentro.br \\ Recebido para publicação: 10/02/2009 - Aceito para publicação: 11/08/2009
}

\begin{abstract}
Resumo
O objetivo deste estudo foi analisar o efeito das emissões de poeira de uma usina de cimento sobre o nível de clorofila de seis espécies arbóreas. Quantificou-se o teor de clorofila mensalmente, durante um ano, em árvores de dois locais distintos: um sob a influência da poeira de cimento e outro distante $50 \mathrm{~km}$ de tal tipo de emissão. Os pigmentos foram extraídos com dimetilsulfóxido, e a sua concentração medida por espectrometria. Araucaria angustifolia, Mimosa scabrella, Ocotea puberula e Schinus terebinthifolius apresentaram concentrações de clorofila mais elevadas no ambiente poluído do que no ambiente-controle, enquanto que Pinus taeda e Matayba elaeagnoides comportaram-se de modo inverso. No local poluído, valores mais elevados em Araucaria, Schinus e Ocotea foram observados durante o inverno, enquanto que Pinus, Mimosa e Matayba tiveram os picos de elevação em meados da primavera. No local livre da poluição, Araucaria e Mimosa mostraram valores uniformes durante todo o ano, enquanto que Ocotea e Matayba tiveram picos de elevação de clorofila em meados do outono e valores reduzidos no inverno. Schinus mostrou picos de depressão no sentido inverno-primavera, e Pinus apresentou picos de elevação na mesma direção.

Palavras-chave: Emissões de cimento; pigmentos foliares; poluição do ar; poluição por cimento; variação sazonal.
\end{abstract}

\begin{abstract}
Chlorophyll content of six tree species under influence of cement dust. The present study aimed to analyse the effect of a cement plant emmissions of dust on chlorophyll content of six tree species. Chlorophyll content was evaluated monthly during a year on trees growing at two different places: one under the influence of cement dust and the other $50 \mathrm{~km}$ far from this type of emmission. Pigments were extracted with dimethylsulphoxide and its concentration measured by spectrometry. Araucaria angustifolia, Mimosa scabrella, Ocotea puberula and Schinus terebinthifolius showed higher chlorophyll concentration at polluted environment in comparison to control, while Pinus taeda and Matayba elaeagnoides performed the inverse manner. Under dust environment, the highest values in Araucaria, Schinus and Ocotea were observed during Winter, while Pinus, Mimosa and Matayba did reach peak values in middle Spring. At the control environment, Araucaria and Mimosa showed uniform values during entire year, while Ocotea and Matayba showed peaks in middle Autumn and decreased values in Winter. Schinus performed reduced values in direction Winter-Spring and Pinus presented level increase in the same direction.

Keywords: Cement emissions; leaf pigments; air pollution; pollution by cement; seasonal variation.
\end{abstract}

\section{INTRODUÇÃO}

Os estudos dos efeitos ecofisiológicos em vegetais devido à interferência que o homem causa ao ambiente natural assumiram nas últimas décadas importância cada vez maior. Devido às dificuldades de controle e as interações múltiplas, não são muitos os estudos desenvolvidos sobre as descargas industriais poluentes e seus efeitos na vegetação. É conhecido que as usinas de cimento emitem poeira que, quando misturada à água, produz um precipitado com elevado $\mathrm{pH}$, tóxico para os seres humanos, animais e vegetais. Segundo observações de Scheffer et al. (1969), nas proximidades dessas indústrias foi verificada uma deposição de partículas da ordem de $1 \mathrm{~g} \cdot \mathrm{m}^{-2} \cdot \mathrm{dia}^{-1}$. As espécies podem apresentar diferentes graus de tolerância à ação da poeira de cimento, como foi comprovado por Iqbal; Shafig (2001). Além disso, os autores relatam que, devido à grande quantidade de cálcio fornecida diariamente, em solos ácidos o $\mathrm{pH}$ é paulatinamente elevado, concluindo que, apesar de ocorrer uma melhora nas propriedades estruturais do solo, observaram um 
abaixamento da disponibilidade do manganês em até 50\%, podendo ocorrer a deficiência desse elemento. Plantas de Schinus terebinthifolius (aroeira-pimenteira) submetidas à poeira de cimento cresceram menos do que as testemunhas, mas apresentam-se como tolerantes ao efeito do cimento (BUJOKAS, 2001). Diminuição do teor total de clorofila e efeitos citotóxicos nas soluções de nutrientes em mudas de Clitoria ternatea submetidas a diferentes concentrações de poeira de cimento foram comprovados por Singh; Shrivastva (2002). Observou-se redução no teor de pigmentos em acículas de Picea abies L. Karst expostas à ação da poeira alcalina de cimento (LEPEDUS et al., 2003), e Cesar et al. (2004) comprovaram que os níveis mais baixos de clorofilas observados em acículas do ano de Picea abies estão relacionados com a necrose das células próximas dos estômatos, provocada pela dissolução das incrustações da superfície das acículas oriundas do depósito de poeira de cimento. Introduzindo poeira de cimento em talhões de Pinus sylvestris à guisa de adubação, Kloseiko; Tilk (2006) comprovaram, após dois meses, que a acidez do solo e os parâmetros fotossintéticos medidos por fluorescência da clorofila não foram afetados pelos tratamentos, mas estes reduziram significativamente a concentração de hexoses em acículas de um ano de idade. Diminuição dos níveis totais de clorofila e da relação a:b, rebaixando a taxa fotossintética e a produção de plantas de oliveira expostas a poeira de cimento foram relatados por Nanos; Ilias (2007). Embora plantas do deserto (Zygophyllum prismatocarpum) tenham sido afetadas pela deposição de poeira, diminuindo o teor de clorofila, inibição da assimilação de $\mathrm{CO}_{2} \mathrm{e}$ do transporte de elétrons, van Heerden et al. (2007) concluem que tais sintomas são reversíveis pela lavagem da poeira provocada pela chuva. Em espécies do cerrado brasileiro, os níveis de clorofila são maiores no início do período seco do que no fim (CARVALHO et al., 2007).

Pelo exposto, destaca-se a importância do estudo das interferências provocadas pela poluição atmosférica nos processos ecofisiológicos dos vegetais. Tais interferências são capazes de afetar, de modo significativo, processos que comandam o crescimento, o desenvolvimento morfológico e os aspectos fitossanitários.

Justifica-se, portanto, que esforços sejam empreendidos no esclarecimento da inter-relação planta-ambiente, principalmente nas regiões próximas às metrópoles, como base para soluções ou alternativas que minimizem o efeito danoso da poluição ambiental, notadamente quando esta implica efeitos cumulativos, colocando em perigo a biodiversidade.

O presente trabalho objetivou estudar as possíveis interferências de uma fábrica de cimento na vegetação situada na região de sua influência, caracterizando a flutuação do nível de clorofila em seis espécies arbóreas durante o período de um ano.

\section{MATERIAL E MÉTODOS}

O presente estudo foi realizado em dois locais distintos, sendo um nas imediações de uma usina de cimento no município de Campo Largo ( $25^{\circ} 27^{\prime} 31^{\prime}$ ' S, 49 $31^{\circ} 42^{\prime}$ ' W, denominado aqui como USINA) e o outro localizado no Centro de Pesquisas de Florestas da Embrapa, no município de Colombo (25'19'16” S, 49 $09^{\prime} 31$ ' W, denominado aqui como EMBRAPA), ambos localizados dentro da Região Metropolitana de Curitiba, estado do Paraná. Partiu-se da premissa de que as plantas localizadas em USINA estariam sob a influência das emissões da usina de cimento. Para efeito comparativo e para servir como controle, assumiu-se que as plantas em EMBRAPA, distando mais de $50 \mathrm{~km}$ da mencionada fonte de emissões, seriam livres de tal influência.

A quantidade de espécies estudadas foi limitada em seis, as quais estavam presentes nos dois locais e apresentavam aspectos externos aproximadamente idênticos. As espécies estudadas foram:

Araucaria angustifolia (Bert.) O. Ktze. (araucária)

Mimosa scabrella Benth. (bracatinga)

Ocotea puberula (Rich.) Ness (canela-guaicá)

Schinus terebinthifolius Raddi (aroeira)

Matayba elaeagnoides Radlkofer (mataíba)

Pinus taeda L. (pinus)

As coletas de material e análise dos pigmentos foram efetuadas mensalmente, durante todo um período vegetativo, entre março de 1996 e fevereiro de 1997. A comprovação de material calcário depositado sobre as folhas foi feita por intermédio de gotas de ácido clorídrico. O material estudado consistiu de amostras coletadas no terço inferior e externo da copa, na sua exposição nordeste a noroeste. Os ramos coletados foram imediatamente colocados em sacos plásticos, hermeticamente fechados e armazenados em caixas de isopor com gelo para o transporte até o laboratório, em Curitiba, PR. Antes da extração, o material foi lavado com água corrente, procurando-se eliminar toda a camada superficial de poeira. A extração foi feita no mesmo dia da coleta, 
adicionando-se dimetilsulfóxido nos tubos de ensaio com os discos de amostras das folhas e deixando-os em banho-maria $\left(65^{\circ} \mathrm{C}\right)$ por 120 minutos. A referência foi baseada no peso fresco de folhas, utilizando-se cinco repetições de amostras compostas oriundas de, no mínimo três árvores de cada espécie. As leituras da absorbância dos extratos foram feitas em espectrofotômetro Metrolab, e os teores de clorofila $a$ e $b$ foram obtidos pelas fórmulas publicadas por Barnes et al. (1992). Para comprovar diferenças causadas pela deposição de material particulado nas folhas, estas foram submetidas à carbonização em mufla, sendo determinado o percentual de cinzas. A comparação entre as médias dessa variável foi feita através do teste t. Para o estudo do efeito estacional sobre o teor de clorofila, foram considerados os valores obtidos nas coletas dos meses de outubro, janeiro, março e julho, representando as estações da primavera, verão, outono e inverno, respectivamente. Foi sobre os dados desses meses que se aplicou a análise da variância e o teste de Tukey ao nível de $95 \%$, para a comparação entre médias. O percentual de participação de cada componente (espécies, locais, estação do ano e interações) na variação total foi calculado utilizando-se os procedimentos propostos por Steel; Torrie (1980).

\section{RESULTADOS}

\section{Teor de clorofila total (clorofila $a+$ clorofila $b$ )}

As plantas apresentaram valores de clorofila diferenciados por espécie, por estação do ano e por local onde foram analisadas. Na tabela 1, dividida em duas partes, apresentam-se os valores médios por mês de coleta, estando em destaque os valores dos meses representativos das estações do ano com a média, seguida do desvio-padrão e significância estatística.

Tabela 1. Teor de clorofila total $\left(\mu \mathrm{g} \cdot \mathrm{mg}^{-1}\right)$ por espécie, por mês e local de coleta.

Table 1. Total chlorophyll content $\left(\mu \mathrm{g} \cdot \mathrm{mg}^{-1}\right)$ by species, by month and place.

\begin{tabular}{|c|c|c|c|c|c|c|}
\hline \multirow{2}{*}{ Mês } & \multicolumn{2}{|c|}{ Araucaria angustifolia } & \multicolumn{2}{|c|}{ Mimosa scabrella } & \multicolumn{2}{|c|}{ Ocotea puberula } \\
\hline & USINA & EMBRAPA & USINA & EMBRAPA & USINA & EMBRAPA \\
\hline Mar96 & $1,37 \pm 0,15 \mathrm{cA}$ & $1,60 \pm 0,15 \mathrm{aA}$ & $4,32 \pm 0,32 \mathrm{cA}$ & $4,48 \pm 0,28 \mathrm{aA}$ & $3,51 \pm 0,25 \mathrm{bB}$ & $4,54 \pm 0,23 \mathrm{aA}$ \\
\hline Abr96 & 1,56 & 1,65 & 4,48 & 4,35 & 3,72 & 4,36 \\
\hline Mai96 & 1,94 & 1,62 & 5,14 & 4,21 & 3,79 & 3,85 \\
\hline Jun96 & 2,27 & 1,61 & 6,60 & 4,13 & 3,94 & 2,97 \\
\hline Jul96 & $2,60 \pm 0,17 \mathrm{aA}$ & $1,68 \pm 0,04 \mathrm{aB}$ & $8,30 \pm 0,90 \mathrm{bA}$ & $4,10 \pm 0,13 \mathrm{aB}$ & $4,09 \pm 0,17 \mathrm{aA}$ & $2,31 \pm 0,08 \mathrm{cB}$ \\
\hline Ago96 & 2,44 & 1,65 & 10,00 & 4,04 & 4,08 & 2,37 \\
\hline Set96 & 2,10 & 1,53 & 11,00 & 4,10 & 4,03 & 2,77 \\
\hline Out96 & $1,96 \pm 0,04 \mathrm{bA}$ & $1,37 \pm 0,06 \mathrm{bA}$ & $11,89 \pm 0,76 \mathrm{aA}$ & $4,09 \pm 0,15 \mathrm{aB}$ & $3,95 \pm 0,26 \mathrm{abA}$ & $3,23 \pm 0,24 \mathrm{bB}$ \\
\hline Nov96 & 1,74 & 1,46 & 10,40 & 3,95 & 3,81 & 3,35 \\
\hline Dez96 & 1,65 & 1,49 & 6,78 & 3,88 & 3,68 & 3,58 \\
\hline Jan97 & $1,12 \pm 0,15 \mathrm{~dB}$ & $1,77 \pm 0,11 \mathrm{aA}$ & $5,11 \pm 0,09 \mathrm{cA}$ & $3,89 \pm 0,24 \mathrm{aA}$ & $2,96 \pm 0,20 \mathrm{cB}$ & $3,68 \pm 0,29 b A$ \\
\hline Fev97 & 1,24 & 1,69 & 4,55 & 4,00 & 3,40 & 3,81 \\
\hline \multirow{2}{*}{ Mês } & \multicolumn{2}{|c|}{ Schinus terebinthifolius } & \multicolumn{2}{|c|}{ Matayba elaeagnoides } & \multicolumn{2}{|l|}{ Pinus taeda } \\
\hline & USINA & EMBRAPA & USINA & EMBRAPA & USINA & EMBRAPA \\
\hline Mar96 & $3,16 \pm 0,28 \mathrm{bA}$ & $2,99 \pm 0,38 \mathrm{aA}$ & $1,96 \pm 0,20 \mathrm{aB}$ & $3,09 \pm 0,05 \mathrm{aA}$ & $1,29 \pm 0,04 \mathrm{bA}$ & $1,49 \pm 0,06 \mathrm{dA}$ \\
\hline Abr96 & 3,05 & 2,96 & 1,98 & 2,88 & 1,31 & 1,62 \\
\hline Mai96 & 3,29 & 2,98 & 1,72 & 2,66 & 1,33 & 2,11 \\
\hline Jun96 & 3,88 & 2,64 & 1,62 & 1,68 & 1,41 & 2,28 \\
\hline Jul96 & $4,2 \pm 0,37 \mathrm{aA}$ & $2,38 \pm 0,11 b B$ & $1,45 \pm 0,09 \mathrm{bA}$ & $1,66 \pm 0,08 \mathrm{cA}$ & $1,43 \pm 0,11 \mathrm{bB}$ & $2,37 \pm 0,25 \mathrm{bA}$ \\
\hline Ago96 & 4,35 & 2,30 & 1,48 & 1,68 & 2,20 & 2,61 \\
\hline Set96 & 4,20 & 2,06 & 1,64 & 1,71 & 3,10 & 2,97 \\
\hline Out96 & $3,61 \pm 0,4 \mathrm{abA}$ & $1,89 \pm 0,15 b B$ & $1,83 \pm 0,22 \mathrm{aA}$ & $1,77 \pm 0,17 \mathrm{cA}$ & $3,40 \pm 0,12 \mathrm{aA}$ & $3,03 \pm 0,21 \mathrm{aB}$ \\
\hline Nov96 & 3,32 & 2.02 & 1,66 & 2,00 & 2,80 & 2,85 \\
\hline Dez96 & 3,19 & 2,57 & 1,36 & 2,55 & 1,84 & 2,47 \\
\hline Jan97 & $3,05 \pm 0,32 \mathrm{bA}$ & $3,23 \pm 0,20 \mathrm{aA}$ & $1,23 \pm 0,19 b B$ & $2,79 \pm 0,11 \mathrm{bA}$ & $1,42 \pm 0,09 \mathrm{bB}$ & $1,73 \pm 0,06 \mathrm{cA}$ \\
\hline Fev97 & 3,18 & 3,18 & 1,52 & 2,95 & 1,30 & 1,58 \\
\hline
\end{tabular}


A seguir são apresentados os valores medidos por espécie, analisando-se a variação observada no teor de clorofila quanto aos locais e mês de coleta, como pode ser observado na figura 1 .

Araucaria angustifolia

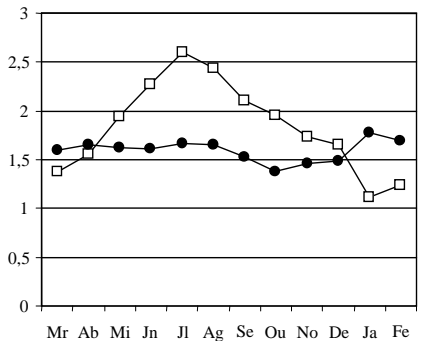

A

Schinus terebinthifolius

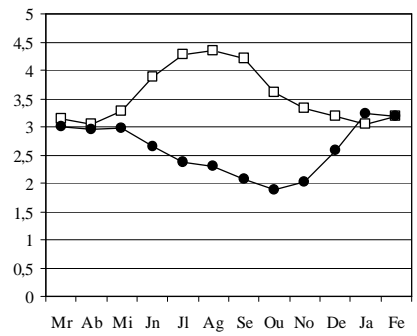

D

- - USINA

Eixo dos X : período de observação - março/96 a fevereiro/97.

Eixo dos $\mathrm{Y}$ : teor de clorofila em micrograma por miligrama.

Figura 1. Variação mensal do teor de clorofila $\left(\mu \mathrm{g} \cdot \mathrm{mg}^{-1}\right)$ das espécies estudadas nos dois locais de coleta (sob influência da poeira de cimento - USINA e livre desta influência - EMBRAPA).

Figure 1. Montly variation of chlorophyll content $\left(\mu \mathrm{g} \cdot \mathrm{mg}^{-1}\right)$ of studied species in relation to place (under the influence of cement dust - USINA and free from such dust - EMBRAPA).

\section{Araucária}

Foi observada uma variação estacional acentuada, assim como efeito significante do ambiente na constituição pigmentária das folhas de araucária, como mostrados na figura 1A. Foram detectadas diferenças significantes entre locais e entre estações do ano. A interação locais $\mathrm{X}$ estações do ano foi também significante. No local USINA, existe uma nítida elevação do teor dos pigmentos nas folhas de araucária no sentido verão-inverno, diminuindo gradativamente em direção à primavera-verão, enquanto que em EMBRAPA essas tendências são quase imperceptíveis. Na variância total, o efeito das estações do ano participou com $46 \%$, ficando a diferença entre locais com $34 \%$.

\section{Bracatinga}

Foi observada uma variação estacional acentuada, assim como efeito significante do ambiente na constituição pigmentária das folhas de bracatinga, como mostra a figura 1B. Foram detectadas diferenças significantes entre locais e entre estações do ano. A interação locais $\mathrm{X}$ estações do ano foi também significante. No local USINA existe uma nítida elevação do teor desse pigmento nas folhas da bracatinga no sentido outono-primavera, diminuindo desse ponto em direção ao verão, enquanto que em EMBRAPA não foi possível detectar qualquer efeito das estações. A maior participação na variância total foi alcançada pelo efeito dos locais, com um percentual de $66 \%$, ficando a diferença entre estações do ano com $25 \%$. Nos meses da primavera, as árvores em USINA mostraram valores até três vezes maiores de clorofila do que as árvores em EMBRAPA nos meses da primavera. 


\section{Canela-guaicá}

Foi observada variação estacional e efeito de local significante na constituição pigmentária das folhas da canela-guaicá, como mostra a figura 1C. Constatou-se existirem diferenças significantes entre os locais e as estações do ano. A interação locais $X$ estações do ano foi também significante. $O$ teor de clorofila das árvores em EMBRAPA foi afetado sensivelmente pelo efeito das estações, conforme mostra o gráfico: os valores tendem a elevar-se no sentido verão-outono, diminuindo daí na direção do inverno e tornando a elevar-se desde o início da primavera em direção ao verão. As árvores sob a ação da poeira de cimento mostraram ligeira elevação no teor de clorofila no sentido do verão até o inverno e caindo daí novamente em direção ao verão. Na variância total, os locais influenciaram em 39\%, enquanto que as estações do ano, $38 \%$.

\section{Aroeira}

Constatou-se variação estacional e efeito de local significante na constituição pigmentária das folhas de aroeira, como mostra a figura 1D. Para o teor de clorofila, apenas os locais influenciaram as avaliações, absorvendo 74\% da variação total. Enquanto que em USINA o teor de clorofila total aumentou nos meses de inverno, em EMBRAPA os pigmentos diminuíram a sua concentração no inverno. A partir do mês de maio, os valores tomam direção oposta, conforme o local de coleta, vindo a nivelar-se somente em pleno verão.

\section{Mataíba}

Constatou-se variação estacional e efeito de local significante na constituição pigmentária das folhas de mataíba, como mostra a figura 1E. Houve diferenças significantes entre os locais e entre as estações do ano, e a interação locais $\mathrm{X}$ estações foi significante. $\mathrm{O}$ teor de clorofila revelou níveis altamente significantes tanto para locais (66\%) como para as estações (26\%). As árvores em USINA mostraram variação estacional sensível, apresentando dois picos de elevação: um no outono e outro na primavera, conforme pode ser visto no gráfico, que também mostra a nítida tendência de diminuição dos pigmentos das árvores de mataíba situadas em EMBRAPA, desde o outono até meados da primavera, quando tornam a elevar-se até o verão.

\section{Pinus}

Foi observada variação estacional e efeito de local significante na constituição pigmentária das acículas de pinus, como mostra a figura 1F. Foram detectadas diferenças significantes entre os locais e entre as estações do ano, e a interação locais X estações foi significante. O teor de clorofila revelou níveis altamente significantes tanto para estações $(81 \%)$ como para os locais de coleta (14\%). As árvores em USINA mostraram variação estacional sensível, apresentando um pico nítido de elevação, iniciando em meados do inverno, atingindo o ponto máximo em meados da primavera e diminuindo daí até o início do verão, conforme mostra o gráfico, onde também é visível a mesma tendência para as árvores situadas em EMBRAPA, sendo que ali a elevação do teor dos pigmentos iniciou mais cedo, em meados do outono, atingindo também seu ponto máximo em meados da primavera.

\section{Teor de cinzas nas folhas e acículas}

Na tabela 2 são mostrados os valores médios do teor de cinzas do material coletado por espécie e por local, que teve a finalidade de comparar os dois locais de coleta.

Tabela 2. Teor médio de cinzas das folhas das espécies estudadas (\% do peso seco).

Table 2. Average leaf ash content of studied species (\% dry weight).

\begin{tabular}{lcccccc}
\hline Local & $\begin{array}{c}\text { Araucaria } \\
\text { angustifolia }\end{array}$ & $\begin{array}{c}\text { Mimosa } \\
\text { scabrella }\end{array}$ & $\begin{array}{c}\text { Ocotea } \\
\text { puberula }\end{array}$ & $\begin{array}{c}\text { Schinus } \\
\text { terebinthifolius }\end{array}$ & $\begin{array}{c}\text { Matayba } \\
\text { elaeaginoides }\end{array}$ & Pinus taeda \\
\hline USINA & 5,70 & 9,16 & 5,05 & 5,97 & 4,34 & 7,59 \\
EMBRAPA & 3,61 & 9,30 & 4,82 & 3,63 & 6,20 & 2,46 \\
Teste t & $* * *$ & n.s. & n.s. & $* * *$ & $* * *$ & $* * *$ \\
\hline
\end{tabular}

***: nas colunas, os valores diferem entre si ao nível de $\mathrm{p} \leq 0,009$. n.s.: nas colunas, os valores não diferem entre si.

As folhas de araucária sob a influência da usina de cimento apresentaram um teor de cinzas 57\% 
mais elevado do que o material existente em EMBRAPA, sendo essa diferença altamente significante. No caso de bracatinga e canela-guaicá, as amostras estudadas não apresentaram diferença significante com referência aos locais de coleta. As folhas de aroeira em USINA continham 65\% mais peso em cinza do que as folhas coletadas em EMBRAPA. A mesma tendência apresentaram as acículas de pinus, com um teor de cinzas três vezes maior em USINA do que em EMBRAPA. No caso de mataíba, ocorreu o inverso do fenômeno, ou seja, as folhas em EMBRAPA continham 43\% mais cinza do que as folhas sob a influência da poeira em USINA.

\section{DISCUSSÃO}

\section{Efeito dos locais de coleta}

Embora não tenha sido realizada uma análise físico-química do ar atmosférico dos dois locais de coleta, a simples observação da deposição de partículas sólidas sobre o limbo das folhas e das acículas serviu para aquilatar o nível de poluição próximo à usina de cimento, fato não constatado no material oriundo das árvores situadas na EMBRAPA. Com exceção da bracatinga-branca e da ocotea-guaicá, que não apresentaram diferenças estatísticas entre os dois locais no que se refere ao teor de cinzas das folhas, nas demais quatro espécies estudadas o material coletado em USINA continha níveis bastante elevados de cinzas quando comparado com material oriundo da EMBRAPA. Parte-se do pressuposto de que existiam diferenças entre os locais de coleta quanto à composição do ar atmosférico, sobretudo no que concerne à deposição de particulados nas folhas.

À parte as interações significantes entre locais e estações do ano que ocorreram em alguns casos, de uma maneira geral os níveis de clorofila foram mais elevados nas árvores localizadas próximas à usina de cimento do que nas árvores localizadas na EMBRAPA. Esse é o caso de araucária, bracatinga-branca, ocotea-guaicá e aroeira-pimenteira, contrastando com os resultados apresentados por Nanos; Ilias (2007) em oliveira. Mataíba-branco e pinus demonstraram valores mais elevados na EMBRAPA do que em USINA, confirmando os efeitos observados em Picea por Lepedus et al. (2003) e por Cesar et al. (2004). Outros tipos de poluição, como, por exemplo, o provocado por dióxido de enxofre, como observado por Bacic et al. (2003) em acículas de abeto, podem acarretar uma redução nos níveis de clorofila. Efeito similar foi verificado por Prajapati; Tripathi (2008) em duas espécies de figueira, mangueira, sissoo, goiabeira e bambu-gigante, pela deposição de poeira em ambiente urbano.

Uma ação direta e deletéria da poeira de cimento sobre a constituição pigmentária das espécies não foi estudada. Os níveis de clorofilas apresentados pelas espécies estudadas são compatíveis com os valores encontrados na literatura. A ação física da poeira, obstruindo estômatos e formando camada limitadora da incidência da radiação solar, como os exemplos citados por Steinhübel (1966), pode ser uma possibilidade plausível para explicar o comportamento das espécies estudadas.

Com a radiação amenizada pela camada de poeira, o mecanismo de síntese de clorofilas pode ter funcionado como no caso de plantas crescendo sob baixa intensidade de luz, ou seja, aumentando a concentração dos pigmentos para compensar a baixa luminosidade, conforme comprovado por Valladares et al. (2005) em plantas de Ilex aquifolium e por Aranda et al. (2005) em mudas de Quercus suber.

As duas coníferas estudadas aqui, a araucária e o pinus, apresentaram níveis de clorofila mais baixos do que as angiospermas, mas não foi possível detectar um efeito deletério da poeira de cimento, conforme constatado por Przemeck (1970) em outras coníferas europeias.

\section{Efeito das estações do ano}

A variação estacional do nível de pigmentos foliares depende de uma série de fatores internos e externos, principalmente temperatura e luz (LINDER, 1972). É conhecido que a temperatura na região metropolitana de Curitiba tende a diminuir nitidamente a partir do outono em direção a meados do inverno e daí elevar-se gradualmente em direção à primavera e verão.

No presente estudo, as diferenças estacionais provocaram comportamentos diferenciados e marcantes no teor de clorofilas das espécies estudadas. De uma forma geral, sob a influência da poeira de cimento, os teores de clorofila aumentaram a partir de meados do inverno em direção à primavera e início de verão. Tal comportamento foi igualmente observado por Demarez et al. (1999) em três espécies arbóreas europeias. Berveiller; Damesin (2008) também constataram valores máximos de clorofila e taxa fotossintética no início do período vegetativo, ou seja, na primavera, em ramos novos de Fagus sylvatica. 
Esses autores relacionaram tais resultados com máxima atividade do rubisco e da PEP-carboxilase.

Por outro lado, as árvores localizadas na EMBRAPA mostraram uma nítida baixa no teor de clorofilas no sentido do outono para o inverno, como no caso de mataíba-branco, ocotea-guaicá e aroeirapimenteira, contrastando com as observações de González et al. (2007) numa rosácea arbórea, que apresentou valores de clorofila mais elevados no inverno do que no verão. A bracatinga-branca e a araucária em EMBRAPA tiveram uma concentração de pigmentos constantes durante todo o período observado. No caso de pinus, observou-se variação estacional similar, tanto nas árvores em USINA como em EMBRAPA, ou seja, com tendência de elevação dos pigmentos desde o inverno até a primavera.

Os resultados mostram ter havido interações entre as condições ambientais (efeito da poeira de cimento) e as condições climáticas (variação de temperatura e radiação). Os vegetais são capazes de produzir clorofila numa amplitude bastante ampla de temperatura do ar. Por outro lado, são sensíveis à luz no que se refere à síntese e destruição das clorofilas. De uma maneira geral, a baixa intensidade de luz estimula o aumento da concentração de pigmentos foliares. Assim, as observações feitas no presente estudo apontam para o aumento da concentração de clorofilas nos meses mais frios e nas árvores sob a influência de particulados, devido à diminuição da radiação, tanto em função da época do ano como em função da camada filtrante proporcionada pela poeira da usina de cimento.

\section{REFERÊNCIAS}

ARANDA, I.; CASTRO, L.; PARDOS, M.; GIL, L.; PARDOS, J. A. Effects of the interaction between dought and shade on water relations, gas exchange and morphological traits in cork oak (Quercus suber L.) seedlings. Forest Ecology and Management, v. 210, n. 1-3, p. 117-129, 2005.

BACIC, T.; UZAREVIC, Z.; GRGIC, L.; ROSA, J.; POPOVIC, Z. Chlorophylls and carotenoids in needles of damaged fir (Abies alba Mill.) from Risnjak National Park in Croatia. Acta Biologica Cracoviensia Series Botanica, v. 45, n. 2, p. 87-92, 2003.

BARNES, J. D.; BALAGUER, L.; MANRIQUE, E. S. S.; DAVISON, A. W. A reappraisal of the use of DMSO for the extraction and determination of chlorophylls a and $b$ in lichens and higher plants. Environ. Exp. Botany, Pergamon, v. 32, p. 85-100, 1992.

BERVEILLER, D.; DAMESIN, C. Carbon assimilation by tree stems: potential involvement of phosphoenolpiruvate carboxilase. Trees - Structure and Function, v. 22, n. 2, p. 149-157, 2008).

BUJOKAS, M. W. O impacto da poeira de cimento em Schinus therebinthifolius Raddi. Curitiba, 2001. Dissertação (Mestrado em Botânica) - Setor de Ciências Biológicas, Universidade Federal do Paraná.

CARVAlho, A. P. F.; BUSTAMAnTe, M. M. C.; KOZOVITS, A. R.; ASNER, G. P. Variações sazonais nas concentrações de pigmentos e nutrientes em folhas de espécies de cerrado com diferentes estratégias fenológicas. Rev. Bras. Bot., v. 30, n. 1, p. 17-25, 2007.

CESAR, V.; LEPEDUS, H.; LJUBESIC, N. Histochemical observations on the needles of Norway spruce trees affected by cement dust pollution. Phyton, v. 44, n. 2, p. 203-214, 2004.

DEMAREZ, V.; GASTELLU-ETCHEGORRY, J. P.; MOUGIN, E.; MARTY, G.; PROISY, C.; DUFRENE, E.; DANTEC, V. L. Seasonal variation of leaf chlorophyll content of a temperate forest. Inversion of the PROPECT model. International Journal of Remote Sensing, v. 20, n. 5, p. 879-894, 1999.

GONZÁlEZ, J. A.; GALlARDO, M. G.; BOERO, C.; LIBERMAN CRUZ, M.; PRADO, F. E. Altitudinal and seasonal variation of protective and photosynthetic pigments in leaves of the world's highest elevation trees Polylepsis tarapacana (Rosaceae). Acta Oecologica, v. 32, n. 1, p. 36-41, 2007.

IQBAL, M. Z.; SHAFIG, M. Periodical effect of cement dust pollution on the growth of some plant species. Turk. J. Bot., v. 25, p. 19-24, 2001.

KLOSEIKO, J.; TILK, M. Influence of cement clinker dust on carbohydrates in needles of Scots pine shortly after the application. Proc. Estonian Acad. Sci. Biol. Ecol., v. 55, n. 2, p. 149-159, 2006.

LEPEDUS, H.; CESAR, V.; SUVER, M. The annual changes of chloroplast pigments content in current- 
and previous-year needles of Norway spruce (Picea abies L. Karst.) exposed to cement dust pollution. Acta Botanica Croatica, v. 62, n. 1. p. 27-35, 2003.

LINDER, S. Seasonal variation of pigmens in needles. A study of Scots Pine and Norway Spruce seedlings grown under different nursery condition. Stud. For. Suec., v. 100, p. 1-37, 1972.

NANOS, G. D.; ILIAS, I. F. Effects of inert dust on olive (Olea europaea L.) leaf physiological parameters. Environmental Science and Pollution Research, v. 14, n. 7, p. 212-214, 2007.

PRAJAPATI, S. K.; TRIPATHI, B. D. Seasonal variation of leaf dust accumulation and pigment content in plant species exposed to urban particulates pollution. Journal of Environment Quality, v. 37, p. 865870, 2008.

PRZEMECK, E. Untersuchungen zur Beurteilung der langfristigen Wirkungen von ZementofenImmisionsstauben auf landwirtschaftlich genutzte Böden. Landw. Forsch., v. 23, p. 204-213, 1970.

SCHEFFER, F.; PRZEMECK, E.; WETZHOLD, P. Zur Kentniss der Wirkung von Zementofenstaubimmisionen auf den Boden. Landw. Forsch., v. 22, p. 326-345, 1969.

SINGH, R. B.; SHRIVASTVA, A. K. Cytotoxic effects and biological damages in Clitoria ternatea by cement kiln dust. Nature, Environment and Pollution Technology, v. 1, n. 4, p. 457-461, 2002.

STEEL, R. G. D.; TORRIE, J. H. Principles and Procedures of Statistics - A Biometrical Approach. New York, McGraw-Hill, Inc., Second Edition, 1980.

STEINHÜBEL, G. Wirkung einer Staubschicht auf die Überwärmung der Blattspreite bei direkter insolation. Biologica-Bratislava, v. 21, p. 277-294, 1966.

VAN HEERDEN, P. D. R.; KRÜGER, G. H. J.; KILBOURN LOUW, M. Dynamic responses of photosystem II in the Namib Desert shrub, Zygophyllum prismatocarpum, during and after foliar deposition of limestone dust. Environmental Pollution, v. 146, n. 1, p. 34-45, 2007.

VALLADARES, F.; ARRIETA, S.; ARANDA, I.; LORENZO, D.; SÁNCHEZ-GOMEZ, D; TENA, D.; SUÁREZ, F.; PARDOS, A. Shade tolerance, photoinhibition sensitivity and phenotypic plasticity of Ilex aquifolium in continental Mediterranean sites. Tree Physiology, v. 25, p. 1041-1052, 2005. 